\title{
Metformin in type 1 diabetes reduces insulin requirements without significantly improving glycaemic control
}

\author{
H. Schatz
}

Received: 15 June 2010 / Accepted: 16 June 2010 /Published online: 9 July 2010

(C) Springer-Verlag 2010

Keywords Glycaemic control - Insulin requirements · Metformin - Type 1 diabetes

To the Editor: In their recent review in Diabetologia [1], Vella et al. identified 197 reports on the effects of metformin added to insulin therapy in type 1 diabetes. Only ten of these studies, including ours [2], involved administration of metformin for at least 1 week, either in random order vs placebo or comparator, or in a crossover design. Nine of the studies, but not ours, specifically mentioned that informed consent was obtained from the patients. For this reason Vella et al. excluded our study from their review. Our paper appeared in 1975, whereas the nine others were published much later: seven appeared between 2000 and 2009, one in 1985 and one in 1987.

Vella et al. concluded from their analysed data that metformin in type 1 diabetes is associated with a reduced insulin-dose requirement, but that there is no clear evidence for an improvement in glycaemic control, judged by $\mathrm{HbA}_{1 \mathrm{c}}$ level. They also found some improvements in lipid profiles with metformin.

Their careful analysis yielded results very similar to those that we had obtained in the course of a 12 week crossover trial with metformin involving 43 children and adolescents with type 1 diabetes, living permanently in a home for patients with diabetes and aged between 3 and 18 years. This study was performed from January to March 1973 and the results were published in 1975 [2].

\footnotetext{
H. Schatz $(\square)$
}

Department of Medicine "Bergmannsheil”, Universitätsklinikum

Bergmannsheil, Ruhr-University Bochum,

Buerkle-de-la-Camp-P1. 1,

44789 Bochum, Germany

e-mail: helmut.schatz@rub.de
One aim of our trial was to determine whether biguanides might improve glycaemic control in type 1 diabetes and, as believed by many diabetologists and pharmacologists at the time, have a 'smoothing effect' on blood glucose fluctuations. We checked blood glucose profiles routinely during the day and counted all hypoglycaemic reactions during day and night. Determination of glycated haemoglobin was not yet available: the increase in glycated proteins in diabetes was first described in 1969 [3] and the use of $\mathrm{HbA}_{1 \mathrm{c}}$ for blood glucose control in diabetes patients was proposed only in 1976 [4]. We found a lowering of the daily insulin dose and a decrease in elevated cholesterol levels, but no improvement of glycaemic control.

While Vella et al. cited our publication from 1975, they excluded it from their evaluation. The reason was that our manuscript did not explicitly mention that all of our patients, and their parents, had been fully informed about the trial and had given their consent. In fact, the patients were eager to receive the 'insulin pills', as they called the metformin tablets, as were their parents (mostly the mothers). At the time of our publication, a statement about informed consent of patients was not yet a prerequisite for acceptance in many national and international journals, nor was it demanded by the editors.

Such an omission of a clinically relevant result that had been carefully established over the relatively long period of 12 weeks in a crossover design benefits neither patients nor the progress of clinical science. We are, however, happy to see that the conclusions reached from our studies in 1975 were confirmed 35 years later by the systematic review by Vella et al. [1].

Duality of interest The authors declare that there is no duality of interest associated with this manuscript. 


\section{References}

1. Vella S, Buetow L, Royle P, Livingstone S, Colhoun HM, Petrie JR (2010) The use of metformin in type 1 diabetes: a systematic review of efficacy. Diabetologia 53:809-820

2. Schatz H, Winkler G, Jonatha EM, Pfeiffer EF (1975) Studies on juvenile-type diabetes in children. Assessment of control under treatment with constant and variable doses of insulin with or without addition of biguanides. Diabete Metab 1:211-220

3. Rahbar S, Blumenfeld O, Ranney HM (1969) Studies of an unusual haemoglobin in patients with diabetes mellitus. Biochem Biophys Res Commun 67:838-843

4. Koenig RJ, Peterson CM, Jones RL, Saudek C, Lehrman M, Cerami A (1976) Correlation of glucose regulation and hemoglobin A1c in diabetes mellitus. New Engl J Med 295:417-420 